\title{
Feature centrality: Naming versus imagining
}

\author{
STEVEN A. SLOMAN \\ Brown University, Providence, Rhode Island \\ and \\ WOO-KYOUNG AHN \\ Yale University, New Haven, Connecticut
}

\begin{abstract}
Being white is central to whether we call an animal a "polar bear," but it is fairly peripheral to our concept of what a polar bear is. We propose that a feature is central to category naming in proportion to the feature's category validity-the probability of the feature, given the category. In contrast, a feature is conceptually central in a representation of the object to the extent that the feature is depended on by other features. Further, we propose that naming and conceptual centrality are more likely to disagree for features that hold at more specific levels (such as is white, which holds only for the specific category of polar bear) than for features that hold at intermediate levels of abstraction (such as has claws, which holds for all bears). In support of these hypotheses, we report evidence that increasing the abstractness of category features has a greater effect on judgments of conceptual centrality than on judgments of name centrality and that other category features depend more on intermediate-level category features than on specific ones.
\end{abstract}

The features of a concept differ in their importance in determining whether an instance is a member of a category. Parentage is more important than appearance in determining whether something is a horse (Keil, 1989), and whether something is made of glass is more important than its shape in determining whether it is called a glass or a cup (Kronenfeld, Armstrong, \& Wilmoth, 1985). We call such scales of relative importance centrality. The main purpose of this paper is to demonstrate that the features that are central for determining the name of an object are not always the features that are central for determining how we think about the object. Naming and thinking about objects impose systematically different demands on the importance that we assign to the objects' various aspects. More specifically, we describe a condition distinguishing features that show convergence in centrality judgments between naming and conceiving from features that show divergence.

Naming has long been known to place special demands on people, demands that are not necessarily reflected in thought. For example, the naming of everyday objects is sensitive to speakers' communicative intent (Brown, 1958; Clark \& Marshall, 1981). The purpose of linguistic cate-

S.A.S. was supported in part by NIMH Grant MH51271 to Barbara Malt and S.A.S. and in part by a grant from Brown University. W.-K.A. was supported by National Science Foundation Grant NSF-SBR 9515085. We thank Barbara Malt and two anonymous reviewers for their comments on a previous draft and Roman Taraban for asking the penetrating question that sparked this research. S. A. Sloman can be contacted at the Department of Cognitive \& Linguistic Sciences, Brown University, Box 1978, Providence, RI 02912 (e-mail: steven_sloman@ brown.edu). W.-K. Ahn can be contacted at the Department of Psychology, Yale University, P.O. Box 208205, New Haven, CT 06520-8205 (e-mail:ahn@pantheon.yale.edu). gories is to help us establish common ground with othersin particular, to identify objects and their properties. This purpose can differ from those of other conceptual tasks. For example, imagining an object might serve a teacher's purpose of highlighting the internal structure of objects of that type. All such purposes could elicit their own, distinct centrality scales. The specific symbols used to represent variables in an equation might be important for naming it, whereas the relations among the variables might be more important for imagining how to solve it. Therefore, we should expect differences between the featural centralities revealed by naming and by nonlinguistic tasks. Indeed, linguistic categorization and nonlinguistic similarity tasks do cause people to put objects together in different ways (Kronenfeld et al., 1985; Malt, Sloman, Gennari, Shi, \& Wang, 1999).

The observation that naming is more closely tied to identifying an object, and conceiving more to the object's internal structure, has led us to the hypothesis that judgments of name centrality and conceptual centrality will converge when the features that identify an instance also play central roles in its internal structure and will diverge when identification features are more peripheral. To measure name centrality, we use judgments of the appropriateness of a name for an object with the target feature missing. To measure conceptual centrality, we use a task that requires access to knowledge not only about an object's features, but also about how those features depend on one another. In particular, we ask people to judge how easily they can imagine an object with the target feature missing. We will show that this task demands the construction of a representation of the object that satisfies the critical structural relations present in the object category. We suspect that people tend to use visual imagery to ac- 
complish the task, but this has no bearing on our hypothesis. We hypothesize that the measures of naming and of conceptual centrality will converge at an intermediate level of categorization - a level often corresponding to Rosch, Mervis, Gray, Johnson, and Boyes-Braem's (1976a) "basic level"-and will diverge at more specific ones. Before spelling out why we predict that these measures will dissociate with varying level of abstraction, we will detail what we mean by naming and conceptual centrality. Subsequently, we will report tests of our hypothesis.

\section{Name Centrality}

The name centrality of a category feature refers to the feature's power to determine the appropriateness of a category label for an object. Having light-colored hair determines whether someone can appropriately be called "blond," and having four doors is central to whether a car can appropriately be called a "four-door sedan." Exceptions can be imagined (a natural blond might dye his or her hair), but the general tendency stands: Cooperative conversation and reference demands that an object referred to by the term blond have, or appear to have, or once have had, or have under some special conditions, light-colored hair.

To obtain estimates of a feature's name centrality, we follow Medin and Shoben (1988) in asking participants about an object that is missing that feature. For example, for the label robin and the feature can fly, we obtain an estimate of name centrality by asking "Suppose an object is in all ways like a robin except that it cannot fly. How appropriate would it be to call this object a robin?"

We propose that a cooperative answer to this question takes into account what a listener will infer if told that this is a robin. Presumably, a listener would infer that the object can fly with a level of certainty approximately proportional to the probability that robins actually can fly. By virtue of providing a label, the speaker is telling a hypothetical listener what to expect of this object. In particular, the speaker is providing probabilistic information about the object's features (cf. Anderson, 1991). So an adequate interpretation of the question is to report a value of name appropriateness that is negatively related to an estimate of the probability of the feature in the category, or what is often called category validity. One way for people to arrive at an estimate of category validity is to generate a sample of instances of category $X$ (e.g., robins) and to estimate the proportion of those instances that have feature $Y$ (e.g., that can $f l y$ ). A representative sample would provide for the most accurate estimates; but people may answer the question by using the availability heuristic (A. Tversky \& Kahneman, 1973), and hence estimates may be biased. In any case, we propose that judgments of name centrality should be correlated with judgments of category validity.

One consequence of our proposal is that name centrality should be highest in the extreme case of a necessary feature. If a feature always obtains in the presence of an object, the feature is necessary for the object, cat- egory validity is perfect, and name centrality judgments should be maximal. Four door should be judged maximally name central for four-door sedans because it is necessary (strictly speaking, a four-door sedan must have four doors). Similarly, if $x$ is an American, $x$ has U.S. citizenship, and so U.S. citizenship should be name central for the category American. In sum, we propose that a feature is central in category naming to the extent that the probability of the feature given the category is high. Below, we test an implication of this proposal.

\section{Conceptual Centrality: Mutability}

Another sense of centrality is conceptual. A feature is central to an object because the feature brings coherence to the mental representation of the object. Having internal organs is central to our concept of mammal because we use internal organs and their relations to other mammalian features to make sense of the life cycle, appearance, and activity of mammals (Keil, 1989). Elsewhere, we have used the term mutability to refer to measures of conceptual centrality (Sloman, Love, \& Ahn, 1998). Features differ by degrees of mutability, the most central being the most immutable. A feature is mutable of an object to the extent that the feature can be mentally transformed without altering the object's mental representation. Tails are mutable with respect to the category of cats because we can easily mentally transform our notion of cat to something that is the same in all respects to cat except that it does not have a tail while we are still thinking about cats.

In previous work, our operationalization of mutability has used a variety of convergent measures. A feature is immutable with respect to an object to the extent that people (1) claim that they would be surprised to encounter the object without the feature; (2) claim that imagining the object without the feature is difficult; (3) rate the object without the feature as atypical of the object category; (4) rate the object without the feature as dissimilar to an ideal instance of the object category; and (5) require time to mentally transform the object to one that does not have the feature (a measure that does not involve a rating). Sloman et al. (1998) and Love (1996) demonstrated convergence among these measures by showing that they were highly correlated. For instance, people's ratings on the following questions about an apple all had roughly equal rankings relative to ratings on analogous questions about other features of apple: (1) How surprised would you be to encounter an apple that did not grow on trees? (2) How easily can you imagine a real apple that does not grow on trees? (3) How good an example of an apple would you consider an apple that does not ever grow on trees? Moreover, the measures did not correlate with other kinds of featural measures. Ratings of centrality did not correlate with ratings of feature salience, such as a feature's degree of prominence (How prominent in your conception of an apple is that it grows on trees?), nor with ratings of feature diagnosticity, like cue validity, the probability of the category given the feature (Of all things that grow on trees, what percentage are apples?). 
In earlier work (Love \& Sloman, 1995; Sloman et al., 1998), we have explored the hypothesis that mutability is a function of a concept's dependency structure. Specifically, we have collected evidence in favor of the hypothesis that a feature is immutable to the extent that other features depend on it. For instance, for the concept chair, the feature has a seat was judged to have many features depending on it, including is comfortable, you sit on it, and holds people. However, people judged that few features of chair depended on is made of wood. In general, measures of degree of dependency correlated highly with immutability judgments: People judged that imagining a chair without a seat is more difficult than imagining one that is not made of wood.

\section{Conceptual Versus Name Centrality}

We have argued that name centrality is a function of feature frequency--in particular, that it is inversely related to category validity - and that conceptual centrality is a function of features' relational structure - in particular, of the extent to which other features depend on the target feature. In most cases, the two types of centrality are highly correlated. After all, a category label should, to some extent, be related to the category's most stable, conceptually central elements. The label bird suggests something that has wings and can fly, and indeed, having wings and flying are relatively immutable properties of birds. If the label did not help to identify such properties, the label would not effectively distinguish birds from categories with different immutable properties. Furthermore, immutable properties sometimes determine what we want to refer to in everyday discourse. In a discussion of the etiology or care of a disease, for example, successful communication sometimes requires that we include reference to whatever we know about the conceptually central aspects of the disease, like the virus that causes it. Whenever conceptual structure orders features in line with category validity, mutability and name centrality will be correlated.

Nevertheless, conceptual and name centrality can be distinguished. In fact, they dissociate every time we use a name that discriminates a category from others that share immutable properties with it. For example, the term polar bear distinguishes one category of bear from others, such as brown bear and black bear, categories that share polar bear's most conceptually central features such as ancestry, physiology, behavioral characteristics, shape, and so forth. But by virtue of specifying a distinct category, the name must refer to one or more features that make the distinction, such as being white. Hence, being white is central to use of the name without being central to our conception of the category.

More generally, features are more likely to be name central but nevertheless mutable when discourse concerns relatively specific categories. At lower levels of abstraction, categories tend to share their dependency structure with other categories. One kind of sedan has much the same dependency structure as another kind of sedan. Four-door sedans differ from two-door sedans, but the differences are not in their most immutable properties. Both have engines, seats, steering wheels, and so forth. The features that are critical for determining whether an object is a four-door sedan or a two-door sedan include one that is necessary without being conceptually central-namely, the number of doors that it has. Similarly, a person is appropriately labeled an "American" if he/she holds United States citizenship. But holding U.S. citizenship hardly describes what it means to be an American. Much more central to the concept American are language, political principles, and cultural icons. Indeed, many Canadians who grew up with stories about George Washington and prime time TV might be thought of as American (in character, not in name). Specific categories are likely to include features that dissociate name centrality from mutability because, by virtue of sharing their dependency structure with other categories, they will contain features that are distinct from those of other categories and yet are not the most conceptually central.

Preliminary support for our hypotheses was reported in Sloman et al. (1998), who showed that increasing the specificity of object categories increased the judged mutability of representative features without affecting their judged appropriateness for determining names. However, that experiment confounded features and categories by using both different categories and different features at intermediate and specific levels. In the present study, we attempted to replicate that finding, this time using the same categories at the two levels and using a pretest to independently choose features at the two levels. We also studied the effect while controlling for category validity. Finally, we investigated our claims about the determinants of conceptual and naming centrality.

\section{Overview of Experiments}

We report three experiments that tested our proposals. Experiment 1 tested the hypothesis that features become less conceptually central at a more specific level of abstraction without becoming less central for naming. Experiment 2 replicated Experiment $l$ in a paradigm which ensured that one of our basic assumptions would hold: namely, that category validity is high for features at both levels. Experiment 3 tested a second of our basic assumptions: that other features depend more on intermediatelevel than on specific features.

\section{EXPERIMENT 1}

The purpose of Experiment 1 was to demonstrate that naming centrality and conceptual centrality are dissociable. More specifically, we predicted that the two kinds of centrality converge at an intermediate level but diverge at a more specific level because conceptual centrality will decrease at the specific level even when naming centrality remains the same. Consider pine trees. The feature has a trunk is more abstract than has needles in the sense that it is true of all trees, whereas the latter dis- 
tinguishes between pine trees and many other kinds of trees, such as maple trees. The intermediate-level feature has a trunk has high naming centrality (the label pine tree is not really appropriate for something that does not have a trunk) and high conceptual centrality (this feature cannot be mutated without changing other aspects of the mental representation of pine trees, such as whether they have bark). In contrast, the specific feature, has needles, has high naming centrality (an object can only tenuously be called a pine tree if it does not have needles) but low conceptual centrality because we can easily mentally transform whether the object has needles while maintaining other features. Hence, the two types of centrality measures are predicted to dissociate when features are diagnostic at the specific level.

In order to empirically determine sufficiently diagnostic target features, a pretest was first conducted. Participants were asked to list a feature that would best distinguish a target category from contrasting categories. The target category was either an intermediate-level category (e.g., tree) or a specific category (e.g., pine tree). The contrasting categories were at the same level as the target category (e.g., flower for the intermediate level and oak tree for the specific level). For each level, the most frequently listed feature was used for the main experiment.

In the main experiment, two types of centrality were measured. Conceptual centrality was measured by asking about the ease with which a feature could be mentally transformed without changing other aspects of the object. Naming centrality was measured by asking about the appropriateness of labeling an object as the target category when it did not have the target feature.

\section{Method: Pretest}

Sixteen Yale undergraduate students participated in the pretest in partial fulfillment of requirements for an introductory psychology course. Each received 12 problems. Six problems involved intermediate-level categories, and 6 involved specific categories subordinate to the intermediate-level categories. Table 1 lists the two sets of categories. For each category, corresponding contrast categories are listed in parentheses. Contrast categories were chosen by the experimenters to be as typical as target categories of their common superordinate. The participants were instructed to write down the attribute or feature that best distinguished the category from its contrast category. For instance, for the first item, the participants were instructed, "Please write down the attribute or feature of birds that best distinguishes them from fish. A bird _____." The order of the 12 problems was completely randomized across the participants. Each problem was presented separately on a computer screen. The participants wrote their responses on a sheet of paper.

\section{Results}

Table 1 lists the most frequently mentioned feature for each category along with its frequency in parentheses. We take these features to be diagnostic of their corresponding category with the following two exceptions, which are due to the particular contrast categories used. For the feature that best distinguishes apples from bananas, is round was the most frequently listed item. However, many typical fruits are also round (e.g., oranges, peaches, melons). Therefore, this feature does not distinguish apples from other fruits. In addition, the most frequently listed feature distinguishing sedans from trucks was is small. Again, this feature does not distinguish sedans from other categories at the same level of abstraction, such as hatchbacks or sports cars. In these two cases, we therefore used the second most frequently listed features in the main experiment. For apple, we used has a core, and for sedan, we used has 4 doors. Otherwise, each of the features appearing in Table 1 was used in the main experiment.

\section{Method: Main Experiment}

Participants. Fourteen Yale undergraduate students participated in the experiment in partial fulfillment of requirements for an introductory psychology course.

Design and Materials. The design of Experiment 1 was a $2 \times 2$ factorial with the level of features (intermediate or specific) and the type of centrality questions (conceptual or naming) as two withinsubjects variables. On the basis of the 12 features selected from the pretest ( 6 intermediate-level and 6 specific), two sets of centrality questions were developed.

For conceptual centrality, the question was in the form "How easy is it to imagine an object that has ALL the features of an X eXcept that it does not have feature $Y$ ?" where $X$ is a target specific category and $Y$ is either a specific or an intermediate-level feature selected from the pretest. Although the features were selected by using both intermediate-level categories (e.g., bird) and specific categories (e.g., robin), only the specific categories were used in the questions in order to avoid any possible confounding that might result from using different categories. For instance, for an intermediatelevel item, the question was "How easy is it to imagine an object that has ALL the features of a robin except that it does not fly?" and

Table 1

Target and Contrast Categories: Experiment 1

\begin{tabular}{|c|c|c|c|c|c|}
\hline \multicolumn{3}{|c|}{ Intermediate Level } & \multicolumn{3}{|c|}{ Specific Level } \\
\hline \multicolumn{2}{|c|}{ Categories } & \multirow[b]{2}{*}{ Features } & \multicolumn{2}{|c|}{ Categories } & \multirow[b]{2}{*}{ Features } \\
\hline Target & Contrast & & Target & Contrast & \\
\hline $\begin{array}{l}\text { bird } \\
\text { tree } \\
\text { apple } \\
\text { car } \\
\text { table } \\
\text { pants }\end{array}$ & $\begin{array}{l}\text { fish } \\
\text { flower } \\
\text { banana } \\
\text { bicycle } \\
\text { chair } \\
\text { shirts }\end{array}$ & $\begin{array}{l}\text { flies }(7) \\
\text { has a trunk }(8) \\
\text { is round }(12) \text { [has a core] } \\
\text { has an engine }(10) \\
\text { has a flat surface }(7) \\
\text { cover the legs }(12)\end{array}$ & $\begin{array}{l}\text { robin } \\
\text { pine tree } \\
\text { red delicious apple } \\
\text { sedan } \\
\text { coffee table } \\
\text { jeans }\end{array}$ & $\begin{array}{l}\text { sparrow } \\
\text { oak tree } \\
\text { golden delicious apple } \\
\text { truck } \\
\text { conference table } \\
\text { dress pants }\end{array}$ & $\begin{array}{l}\text { has a red breast (9) } \\
\text { has needles (9) } \\
\text { is red }(10) \\
\text { is small }(7) \text { [has } 4 \text { doors] } \\
\text { is short }(12) \\
\text { are made of denim (8) }\end{array}$ \\
\hline
\end{tabular}

Note-Most frequent feature for each category is shown along with frequency of mention in pretest (in parentheses). Features shown were used in main experiment except in two cases where bracketed feature was used instead. 
for a specific item, the question was "How easy is it to imagine an object that has ALL the features of a robin except that it does not have a red breast?"

For naming centrality, the question was in the form "Suppose an object is in all ways like an X except that it does not have feature $\mathrm{Y}$. How appropriate would it be to call this object an X?" As in the conceptual centrality questions, only the specific categories were used but the features varied in level.

In addition to the 12 items for each type of centrality question, 8 filler items were added to encourage participants to use the full range of the response scale. These filler items were developed by using four categories with two features that were expected to be neither name central nor conceptually central. The following categories and associated features were used: beagle - enjoy swimming, 13 inches tall; IBM - price increasing every year, shows color images; bicycles - light weight, 10-speeds; tomatoes-tastes good, sold in a supermarket.

Procedure. Seven participants received the naming centrality questions followed by the conceptual centrality questions, and $7 \mathrm{re}$ ceived them in the opposite order. The groups showed no systematic differences, and so the results are collapsed across them. Within each task, the order of the 20 problems was randomized for each participant.

For the naming centrality task, the participants were instructed as follows:

In this section, we will ask you to evaluate the appropriateness of a label. Sometimes, in conversation, objects seem to require certain attributes to warrant a specific label. It would be inappropriate when discussing someone, for example, to call a man a "bachelor" if he was known to have a spouse. We'd like to know what properties you think are necessary to apply a label to an object. Your task is to rate how appropriate a label is for an object that is missing a specified property. We will ask you questions like:

A. Suppose an object is in all ways like a house except that it does not have walls. How appropriate would it be to call this object a house?

The questions were presented by computer, and the participants responded by typing a number between 1 (very inappropriate) and 9 (very appropriate) on the computer keyboard.

For the conceptual centrality task, the participants received the following instructions:

In this section, we will ask you to evaluate the ease with which you can transform an image of an object. We'll ask you to imagine an ideal object, and then change some specified part or aspect it. For example, imagine a door, then transform it in your mind into a door without a doorknob. We would like to know how easily you can complete this transformation. Your task, then, is to rate the ease of the transformation required to get from the original to the mutated form. We will ask you questions like:

A. How easy is it to imagine an object that has all the features of a guitar except that it does not have a neck?
The participants responded by typing a number between 1 (very easy) and 9 (very difficult).

For both tasks, two other examples were provided and discussed. The instructions emphasized that the experiment concerned real category members, not metaphorical, broken, or toy ones. The participants could correct their answer before preceding to the next question. The experiment was programmed using PsyScope 1.1 (Cohen, MacWhinney, Flatt, \& Provost, 1993).

\section{Results and Discussion}

As predicted, naming centrality did not differ between intermediate-level and specific features. However, conceptual centrality did. Table 2 shows the mean ratings for each centrality measure for each item at each level, as well as the overall means. The name centrality and the mutability scales used in the experiments are in the opposite directions in that 1 in the name centrality scale means the feature is very central whereas 1 in the mutability scale means the feature is peripheral. To facilitate presentation of the results, the name centrality scale was reversed so that the two scales had the same directionality. In Table 2, the higher the number is, the more central the feature is.

A repeated measures analysis of variance was conducted with task (conceptual or naming) and level (specific or intermediate-level) as two factors. Consistent with the hypothesis, there was a reliable interaction between the two factors $\left[F(1,13)=10.53, M S_{\mathrm{e}}=0.80, p<.01\right]$. There was also a reliable main effect of level $[F(1,13)=$ $\left.4.88, M S_{\mathrm{e}}=1.41, p<.05\right]$, because the specific features were judged to be less conceptually central than the intermediate-level features. Indeed, post hoc analyses show that the difference between the two levels occurred only with respect to conceptual centrality. The intermediatelevel features had reliably higher conceptual centrality than did the specific features $[t(14)=3.61, p<.01]$, whereas the two features did not differ with respect to naming centrality $(p=.86)$. In addition, there was a reliable main effect of task $\left[F(1,13)=19.99, M S_{\mathrm{e}}=1.92\right.$, $p<.01]$, because name centrality judgments were higher than conceptual centrality ones.

Another repeated measures analysis of variance was conducted, with the six items as a random variable. Again, there was a reliable interaction effect between task and level $\left[F(1,5)=12.53, M S_{\mathrm{e}}=0.29, p<.05\right]$. There was also a reliable main effect of task $\left[F(1,5)=78.23, M S_{\mathrm{e}}=0.21\right.$,

Table 2

Mean Ratings of Two Types of Centrality for Two Levels of Features for Six Items: Experiment 2

\begin{tabular}{lccccc}
\hline & \multicolumn{2}{c}{ Conceptual Centrality } & & \multicolumn{2}{c}{ Naming Centrality } \\
\cline { 2 - 3 } \multicolumn{1}{c}{ Category } & Intermediate & Specific & & Intermediate & Specific \\
\hline Robin & 3.79 & 2.86 & & 4.86 & 4.64 \\
Pine tree & 6.00 & 4.14 & & 6.50 & 6.21 \\
Red delicious apple & 4.64 & 3.57 & & 5.93 & 7.14 \\
Sedan & 3.93 & 3.07 & & 5.21 & 5.79 \\
Coffee table & 3.86 & 3.29 & & 5.21 & 4.79 \\
Jeans & 6.07 & 2.50 & & 5.86 & 5.43 \\
Average & 4.71 & 3.24 & & 5.60 & 5.67 \\
\hline
\end{tabular}


$p<.001]$, and a marginally reliable main effect of level $\left[F(1,5)=5.30, M S_{\mathrm{e}}=0.56, p=.07\right]$. As can be seen in Table 2, for all six items, conceptual centrality decreased between intermediate and specific levels. For naming centrality, however, mean ratings remained roughly the same (coffee table, jeans, pine tree, robin) or even went up (red delicious apples, sedan) as feature level changed.

One of the category-feature pairs in this experiment (red delicious apple/is red) is unique in that the feature is part of the category label. A glance at Table 2 reveals that the effect is not driven by this one item. Nevertheless, this pair may be special in that denying a feature that is part of the name might have a larger effect on naming than on imagining. We defer further discussion of this issue to the results of Experiment 2.

In sum, in Experiment 1, measures of conceptual and naming centrality diverge more at specific levels of feature abstraction than at more abstract levels. This replicates Sloman et al.'s (1998) finding that conceptual and naming centrality are dissociable. Unlike in Sloman et al.'s experiment, the result was obtained when holding the category label constant across the two levels while varying the target feature.

\section{EXPERIMENT 2}

Our hypothesis is that the dissociation between conceptual and naming centrality across intermediate-level and specific features will occur with features that distinguish categories at each level. We expect features that distinguish a specific category from other specific categories (as having a red breast distinguishes robins from other birds) to have high naming centrality despite not being conceptually central. One prerequisite for a feature to distinguish a category from others is that it have high category validity in the target category. That is, the probability of the feature among instances of the category should be high. In Experiment 1, we simply assumed that the category validity of features was high. Experiment 2 was an attempt to replicate Experiment 1 while checking this assumption. Each participant was asked to judge not only the conceptual and name centrality of each category feature but also its category validity. Our analysis was restricted to items showing appropriate levels of category validity; that is, all data will be conditionalized on high category validity judgments. Experiment 2 was also an attempt to replicate Experiment 1 with a different set of stimuli.

\footnotetext{
Method

Participants. Six undergraduates from Brown University participated in the experiment and received $\$ 6$ per hour for their participation in this and another unrelated experiment. In addition, 13 residents of the New Haven area participated and received \$7 for participation in this and other unrelated experiments.

Materials and Design. Seven categories were used; they are shown in Table 3 along with the features tested. We chose categories and associated features that met the following requirements: The category had to be sufficiently specific to share a dependency
}

structure with other categories; the categories chosen should constitute a varied set that included natural kinds and artifacts; also, the feature set available had to include features from multiple levels of abstraction because the relevant comparisons involved multiple levels. We obtained such categories and features from two sources: (1) Three categories and corresponding feature sets (green seedless grapes, claw hammer, and knit shirt) were selected from Rosch, Mervis, Gray, Johnson, and Boyes-Braem (1976b), who compiled a set of features for categories by asking undergraduates to write down attributes that they could think of for each category and then eliminating those that were not commonly listed. (2) Three category-feature sets (cucumber, pine tree, and robin) were selected from Dean and Sloman (1995), who compiled features by first asking a group of Brown University students to write down attributes that they could think of for a number of categories and then asking another group to rate each category for the extent to which it possessed each feature. Features given low judgments or judged irrelevant to the category by the second group were eliminated.

We classified the features of each category into three levels. If a feature obtained for a very general category of instances (e.g., is edible is true of the general category fruit), we classified it as a superordinate feature and ignored it in this experiment. If a feature held at an intermediate level, but not for its superordinate category, we labeled it "intermediate-level" (e.g., grows on a vine for the intermediate-level category grapes). Finally, if a feature held only at a relatively specific level, we classified it as a "specific" feature (e.g., are green for green seedless grapes). The Appendix shows the full set of features along with each feature's assigned level of abstraction. We chose one intermediate-level feature and one specific feature from each feature set that appeared to have high category validity.' Nine filler items were used in this experiment: eight from Experiment 1 plus an additional one.

Procedure. The participants were asked three sets of questions about these 14 features, one each concerning conceptual centrality, naming centrality, and category validity. The conceptual and naming centrality questions had the same formats as in Experiment 1. Category validity questions were in the form "What percentage of X's have feature Y?"' Filler items were included in each of the three tasks.

The instructions given for the conceptual and naming centrality tasks were identical to those of Experiment 1 . For the category validity task, the participants were instructed to estimate the percentage of category instances, between 0 and 100 , that had the target feature. The order of the three tasks was counterbalanced across participants. There was no effect of order, so it is ignored in the analyses below. In other respects, the procedure was identical to that of Experiment 1.

\section{Results and Discussion}

Our prediction was that the features with high category validity at the specific level would show the dissociation relative to features with high category validity at the intermediate level. Our hypothesis did not apply to features with low category validity, so such features were

Table 3

Features and Categories: Experiment 2

\begin{tabular}{lll}
\hline \multirow{2}{*}{ Category } & \multicolumn{2}{c}{ Level of Feature } \\
\cline { 2 - 3 } Claw hammer & has a head & pulls nails \\
Cucumber & has seeds & is green \\
Green seedless grapes & are juicy & are green \\
Knit shirt & covers the torso & stretches \\
Pine tree & has bark & has pointy needles \\
Robin & has wings & has a red breast \\
\hline
\end{tabular}


omitted from analysis of centrality. Specifically, all judgments reported are conditionalized, for each participant, on features for which that participant gave a category validity judgment of at least $85 \%$. This eliminated $17.1 \%$ of the judgments-mainly those for robin.

Table 4 shows mean ratings for each item for each centrality task for each level of features as well as overall means. A repeated measures analysis of variance across participants was conducted with task (conceptual or naming) and level (specific or intermediate-level) as two factors. Once again, as predicted, the interaction between the two factors was highly significant $[F(1,19)=$ $\left.11.92, M S_{\mathrm{e}}=.71, p<.01\right]$, because the difference between the intermediate-level and the specific features was greater for conceptual centrality (with a mean difference of 0.94) than for naming centrality (with a mean difference of 0.35 in the opposite direction). Post hoc analyses showed that the conceptual centrality of specific features was reliably lower than that of intermediatelevel features $[t(19)=2.49, p<.05]$, but the specific and the intermediate-level features did not differ with respect to naming centrality $[t(19)=1.07$, n.s.]. The main effects of task $\left[F(1,19)=2.37 ; M S_{\mathrm{e}}=2.16\right]$ and of level $\left[F(1,19)<1 ; M S_{\mathrm{e}}=1.84\right]$ were not significant. The expected interaction turned out to be robust in the sense that it occurred whether or not we conditionalized on category validity judgments. Without conditionalizing, the interaction between the two factors was still significant $\left[F(1,19)=11.78, M S_{\mathrm{e}}=0.42, p<.01\right]$ and in the expected direction. Again, no reliable main effect of task $\left[F(1,19)=1.82, M S_{\mathrm{e}}=2.11\right.$, n.s. $]$ or of level $[F(1,19)=$ $2.75, M S_{\mathrm{e}}=0.88$, n.s.] obtained. However, in this experiment, the effects were not statistically significant across our six items $\left[F(1,5)=1.77, M S_{\mathrm{e}}=1.18\right.$, n.s.]. As predicted, naming centrality judgments showed no systematic pattern; however, they did vary widely. Conceptual centrality judgments all showed the predicted effectexcept for one, pine tree. Unlike judgments on this item in Experiment 1, its specific feature, has pointy needles, was judged even more conceptually central than the intermediate-level feature, has bark.

Like Experiment 1, Experiment 2 included a single category-feature pair having the feature as part of the

Table 4

Mean Conceptual Centrality and Naming Centrality Judgments for Intermediate-Level and Specific Features for Six Categories: Experiment 2

\begin{tabular}{lcccc}
\hline & \multicolumn{3}{c}{ Type of Centrality } \\
\cline { 2 - 5 } \multicolumn{1}{c}{ Category } & \multicolumn{2}{c}{ Conceptual } & \multicolumn{2}{c}{ Naming } \\
\cline { 2 - 5 } \cline { 5 - 5 } & Intermediate & Specific & Intermediate & Specific \\
\hline Claw hammer & 7.11 & 5.42 & 7.22 & 6.53 \\
Cucumber & 4.56 & 4.40 & 4.28 & 5.05 \\
Green seedless grapes & 5.31 & 4.32 & 3.92 & 8.37 \\
Knit shirt & 5.27 & 4.31 & 5.21 & 3.85 \\
Pine tree & 6.05 & 6.83 & 6.10 & 6.61 \\
Robin & 6.61 & 4.00 & 7.00 & 4.75 \\
Average & 5.90 & 4.96 & 5.76 & 6.11 \\
\hline
\end{tabular}

name (green seedless grapes/are green in this case). The data suggest that denying the feature of this category had a particularly large effect on naming centrality judgments, suggesting that naming is highly sensitive to the presence of features referred to explicitly by the object label. This finding cannot account for the interaction between categorization task and level of specificity, however. If we combine the data from Experiments 1 and 2 but omitting the two categories whose specific feature is referenced in the name (red delicious apple and green seedless grapes), the pattern of results remains identical to that reported for each experiment separately. In particular, the interaction between task and level remained significant both by participants $\left[F(1,33)=4.59, M S_{\mathrm{e}}=1.21\right]$ and by items $\left[F(1,9)=6.84 ; M S_{\mathrm{e}}=.27\right.$; both $\left.p \mathrm{~s}<.05\right]$.

In sum, Experiment 2 showed the same pattern of results as did Experiment 1 of this paper and Experiment 4 of Sloman et al. (1998) in a situation in which an underlying assumption of our hypothesis was verified; namely, the judged category validity of features was high. However, in this experiment, the results were statistically significant only across participants, and not across items. Because we used only 6 items in this experiment, it did not possess a lot of power to detect an item difference. Nevertheless, the fact that differences were in the expected direction, and that significant effects have been obtained with a variety of items in previous studies gives us some confidence that the results can be safely generalized across items of the type used here.

\section{EXPERIMENT 3}

Experiments 1 and 2 have demonstrated that features that discriminate specific categories have lower conceptual centrality than do features that discriminate intermediate-level categories, even though the features' name centrality and category validity do not differ. We predicted this effect on the hypothesis that specific category features with high category validity would have low conceptual centrality relative to intermediate-level features because few other features would depend on them in a conceptual representation of the category, whereas many other features would depend on intermediate-level features with high category validity. We predicted that little difference would obtain for name centrality because, rather than being a function of dependency, namecentrality is more closely related to category validity, and category validity was not systematically varied in the experiments.

Experiment 3 tested the assumption that more features depend on intermediate-level features than specific features using a measure of categories' dependency structures. We used the features described in the Method section of Experiment 2. Note that these features were collected for reasons entirely distinct from the present concerns and thus provide an independent and unbiased set to test the present hypothesis. We expect to show, for instance, that other features of green seedless grapes depend more on is juicy (an intermediate-level feature) than 
on is green (a specific one). Participants were given a list of features for each category and were asked to draw arrows indicating which features depend on which other features.

\section{Method}

Participants. Fifteen undergraduates at the University of Louisville participated in the task in partial fulfillment of the requirements of an introductory psychology course, and 20 students at Brown University participated in return for compensation at a rate of $\$ 6$ per hour.

Materials. We tested the categories used in Experiment 2. Green seedless grapes, claw hammer, and knit shirt were tested with the University of Louisville students, and the categories robin, pine tree, and cucumber were tested with the Brown students. The features listed in the Appendix were used.

Procedure. For each category, the participants received a list of features arranged in a circle on a sheet of paper. They were instructed to draw arrows indicating which features depended on which other features. The participants also received several examples in order to explain what we meant by "depends on." For instance, they received an example using the object house: The feature has windows would depend on the feature has walls, because windows cannot exist without walls. The participants were also instructed that features can depend on each other (e.g., having a roof depends on having walls, and having walls can depend on having a roof because a roof is needed to cover the walls). They were also instructed that sometimes a feature can depend on more than one feature (e.g, having ceilings can depend on having walls and having a roof). Finally, they were told to indicate the strength of the dependency by putting a number by each arrow. The strength of the dependency was given on an a 1-7 scale $^{2}$ anchored at its minimum with very weak dependency and its maximum with very strong dependency. ${ }^{3}$

\section{Results}

For each feature for each subject, the mean dependency rating was calculated by summing the degree to which other features depended on the target feature and dividing it by the number of features for that category minus one (i.e., the total number of possible features that could depend on the target feature). The value 0 was used when no dependency was drawn by the participants. Ratings were normalized by dividing them by the maximum value on the rating scale. Hence, if a feature was not depended on by any other feature, the mean dependency rating would be 0 . If a feature was strongly depended on by all other features in the same category, the mean dependency rating would be 1 . The means for each level for each category are reported in Table 5.

Mean dependency ratings for each feature level were subject to a repeated measures analysis of variance. There was a highly significant effect of level $[F(2,68)=$ $\left.12.02, M S_{\mathrm{e}}=.00052, p<.0001\right]$. In particular, specific features had fewer dependencies than intermediate-level features $[t(34)=4.02, S E=0.0047, p<.001]$. These differences were also significant across the six items; both the overall effect $\left[F(2,10)=9.74, M S_{\mathrm{e}}=.00012\right.$, $p<.01]$ and the comparison of specific to intermediatelevel features $[t(5)=4.84, S E=0.0040, p<.01]$ were significant. Because we had no predictions on this issue, we merely note that superordinate features turned out to have roughly the same number of dependencies as did
Table 5

Mean Normalized Dependency Measures for Features of Six Categories at Each of Three Levels of Abstraction

\begin{tabular}{lccc}
\hline & \multicolumn{3}{c}{ Level of Features } \\
\cline { 2 - 4 } \multicolumn{1}{c}{ Category } & Superordinate & Intermediate & Specific \\
\hline Claw hammer & 0.063 & $0.080(0.101)$ & $0.044(0.061)$ \\
Cucumber & 0.043 & $0.039(0.039)$ & $0.032(0.023)$ \\
Green seedless grapes & 0.074 & $0.042(0.052)$ & $0.021(0.012)$ \\
Knit shirt & 0.078 & $0.049(\mathrm{~N} / \mathrm{A})$ & $0.035(0.015)$ \\
Pine tree & 0.035 & $0.032(0.054)$ & $0.012(0.019)$ \\
Robin & 0.028 & $0.030(0.054)$ & $0.011(0.0054)$ \\
Average & 0.051 & $0.044(0.061)$ & $0.025(0.022)$
\end{tabular}

Note-Dependency values for particular features used in Experiment 2 appear in parentheses. Data are from Experiment 3.

intermediate-level features $[t(34)=1.16, S E=0.0062$, n.s.]. The difference in degree of dependency held for the particular set of features used in Experiment 2, whose mean dependency ratings also appear in Table 5 . Considering only those features, specific features had fewer dependencies than intermediate-level ones both by participants $[t(34)=3.67, S E=0.0099]$ and by items $[t(4)=$ 6.67, $S E=0.0054$; both $p s<.01]^{4}$

In sum, Experiment 3 strongly supports our contention that other features depend more on intermediate-level features than on specific ones. This difference could account for the corresponding difference in conceptual centrality between the two types of features.

\section{GENERAL DISCUSSION}

In summary, the data support two tenets of our proposal: First, judgments of conceptual centrality-as measured by ease-of-imagining judgments-were greater for category features at a more abstract level than for more specific features, a difference that did not obtain for judgments of name centrality - as measured by the complement of name appropriateness judgments. Second, features of more abstract categories had more other features depending on them than did features of more specific categories.

These results, together with our previous demonstrations that the conceptual centrality of a feature is correlated with the number of other features that depend on it (Sloman et al., 1998), sit comfortably with our proposals about category centrality. We propose that conceptual centrality reflects the place of a feature in an internal representation. A feature is conceptually central to the extent that it binds the category representation --that is, to the extent that other features depend on it. In contrast, a feature is name central to the extent that it has high category validity - that is, to the extent that it obtains when an instance of the name category does. Because these notions of centrality are different, measures of the two should be dissociable, as they were in Experiments 1 and 2 and in Sloman et al. (1998).

We argued above that the name centrality of a feature for a category should be proportional to category valid- 
ity, the probability of the feature in the category, on the assumption that people will deem a feature critical for determining a name in proportion to the feature's relative frequency in the name category. Specifically, people will judge that an object missing the critical feature should not be called by a name to the extent that objects that have that name also have that feature. Of course, our hypothesis is task specific. If the naming task were different, a different model would be appropriate. For instance, if we were concerned with how participants chose a name for an object, category validity might not be the right model. Cue validity, the probability of the category given features of the object, might be a better model of such a task because it would indicate the degree to which the feature picked out the category from others. However, choosing a name is well known to be highly context sensitive (e.g., A. Tversky, 1977). Therefore, a model of such a task that takes into account not only the cue validity of a feature for the particular name category of interest but also the cue validity of the feature for other contextually relevant categories would be even better. Similarly, the name centrality task that we have studied here is likely to be context sensitive, and so an adequate model of it might well have to take into account the category validity of the critical feature of other contextually relevant categories too.

\section{A Definition of the Basic Level?}

One implication of our argument is that the most specific level of abstraction at which name and conceptual centrality are most likely to converge is the level at which most of a category's discriminating features are central parts of the category's dependency structure. This is the level at which the members of a category's contrast set have their own rich, yet distinct dependency structures. For example, the default contrast class of the category bear includes-for most Americans-such categories as deer, moose, and wolf. These contrasting categories have noticeably different dependency structures than bear: category instances look different, move differently, have different habits, and so on. So we can choose a feature to pick out bears that not only discriminates bears from members of other categories, but also is central to our concept of bears. We speculate that this level of maximal convergence is at the same level of abstraction that Rosch et al.'s (1976a) measures picked out and that they called the "basic level" of categorization. This speculation dovetails with B. Tversky and Hemenway's (1984) proposal that the basic level of object categorization is the highest level at which categories share parts. Their hypothesis finds additional support in the fact that most of our discriminating features turned out to be object parts. However, not all were, and so the possibility remains open that other nonphysical features are also crucial in determining people's preferred level of reference.

We choose the basic level because it is the level that shows the most differentiation (Rosch et al., 1976a): Category members are most similar to one another and most dissimilar to members of other categories. However,
Markman and Wisniewski (1997) have shown that basiclevel categories share a lot of representational structure; they are "alignable." For example, bears and deer have many differences, but the most salient differences are on the same dimensions: Even though they move differently, they both move; even though their body parts have different shapes, they are homologous. This contrasts with categories from different superordinates. Bears and screwdrivers not only have many differences, but the differences are incomparable. In sum, Markman and Wisniewski demonstrate that basic-level categories share representational structure with other basic-level categories from the same superordinate; superordinate categories do not share representational structure with each other. More critical to our claim, Markman and Wisniewski also demonstrate that basic-level categories with a common superordinate differ with each other on alignable dimensions; that is, their representational structures are alignable, but their features are different.

Thus, we agree with Markman and Wisniewski (1997) that the basic level might be usefully defined as the most specific level at which categories with a common superordinate have distinct dependency relations between features, because their features contrast with those of other categories at the same level. This would explain why categories are relatively easy to process at the basic level: The convergence of dependencies would elicit a representation most naturally at that level, because the representation would accrue structural support from the large number of dependencies and that support would be relatively unique to that representation. Because of the convergence of dependencies, the representation would not have to compete with others. Moreover, the agreement between name and conceptual centrality at this level would allow each to provide mutual support for the other. When they diverge, as they can at the subordinate level, language and conception would conflict, which could interfere with processing. Referring to a " 1956 dime" rather than just a "dime" is confusing if I am discussing a financial transaction involving 10 cents. The advantage of the basic over the superordinate level may arise from the limited structure of superordinate concepts. Very abstract (superordinate) categories tend not to have much dependency structure, because they have very few features (consider how few features are shared by all animals or by all furniture).

\section{Implications}

A debate has raged in philosophy (e.g., Austin, 1961; Putnam, 1975; Wittgenstein, 1953) and in psychology (e.g., Armstrong, Gleitman, \& Gleitman, 1983; Malt, 1994) about what determines the meaning and use of a concept. Two key ideas that have emerged from this debate are that concepts have "theoretical" cores (Keil, 1989; Murphy \& Medin, 1985) and that concepts are attributed "essences" even when the essential properties are unknown (Medin \& Ortony, 1989). The implication of our work for this debate is that the centrality of a cat- 
egory's features is relative to the function being served by the category. Concepts have multiple facets. The importance of a feature depends not only on the identity of the feature and its relation to other conceptual features, but also on the goal of the agent using the concept.

The necessity of distinguishing name and conceptual centrality points to a gap between the uses of concepts in language and thought (Brown, 1958). Conceptual structure must be viewed from one perspective to account for linguistic usage and a different one to account for thought. If the hypothesis that we have presented here is correct, labeling depends more on knowledge about the relative frequency of features in a category, and less on knowledge about the relations between features. This may be so because relative frequency information generally can be obtained with more confidence and more reliability than relational information and is thus more easily shared between individuals. A representational system that maintains relative frequency information need only keep statistics on each feature of each category. But maintaining relational information requires keeping statistics on (at least) every pair of features of a category, a vastly greater amount of data to keep track of.

Finally, the need to distinguish these two forms of categorization suggests that other forms may require their own special analyses. For example, object recognition almost surely comprises a different set of processes than does the kind of thoughtful deliberation that a jury engages in (one hopes) when trying a court case (cf. Sloman, 1996).

\section{REFERENCES}

ANDERSON, J. R. (1991). The adaptive nature of human categorization. Psychological Review, 98, 409-429.

armstrong, S. L., Gleitman, L., \& Gleitman, H. (1983). What some concepts might not be. Cognition, 13, 263-308.

Austin, J. L. (1961). Philosophical papers. Oxford: Oxford University Press.

Brown, R. (1958). How shall a thing be called? Psychological Review, 65, 14-21.

Clark, H. H., \& Marshall, C. R. (1981). Definite reference and mutual knowledge. In A. K. Joshi, B. Webber, \& I. A. Sag (Eds.), Elements of discourse understanding (pp. 10-63). Cambridge: Cambridge University Press.

Cohen, J. D., MacWhinney, B., Flatt, M., \& Provost, J. (1993). PsyScope: An interactive graphic system for designing and controlling experiments in the psychology laboratory using Macintosh computers. Behavior Research Methods, Instruments, \& Computers, 25, 257-271.

DEAN, W., \& Sloman, S. A. (1995). A connectionist model of semantic memory. Unpublished manuscript.

KeIL, F. (1989). Concepts, kinds, and cognitive development. Cambridge, MA: MIT Press.

Kronenfeld, D. B., Armstrong, J. D., \& WiLmoth, S. (1985). Ex- ploring the internal structure of linguistic categories: An extensionist semantic view. In J. W. D. Dougherty (Ed.), Directions in cognitive anthropology (pp. 91-113). Urbana: University of Illinois Press.

LOVE, B. C. (1996). Mutability, conceptual transformation, and context. In Proceedings of the Eighteenth Annual Conference of the Cognitive Science Society (pp. 459-463). Mahwah, NJ: Erlbaum.

Love, B. C., \& Sloman, S. A. (1995). Mutability and the determinants of conceptual transformability. In Proceedings of the Seventeenth Annual Conference of the Cognitive Science Society (pp. 654-659). Hillsdale, NJ: Erlbaum.

Malt, B. C. (1994). Water is not $\mathrm{H}_{2}$ O. Cognitive Psychology, 27, $41-70$.

Malt, B. C., Sloman, S. A., Gennari, S., Shi, M., \& Wang, Y. (1999). Knowing versus naming: Similarity and the linguistic categorization of artifacts. Journal of Memory \& Language, 40, 230-262.

Markman, A. B., \& WisNiewski, E. J. (1997). Similar and different: The differentiation of basic-level categories. Journal of Experimental Psychology: Learning, Memory, \& Cognition, 23, 54-70.

Medin, D. L., \& Ortony, A. (1989). Psychological essentialism. In S. Vosniadou \& A. Ortony (Eds.), Similarity and analogical reasoning (pp. 179-195). New York: Cambridge University Press.

MEDIN, D. L., \& ShOBEN, E. J. (1988). Context and structure in conceptual combination. Cognitive Psychology, 20, 158-190.

MuRPHy, G. L., \& MEdin, D. L. (1985). The role of theories in conceptual coherence. Psychological Review, 92, 289-316.

Putnam, H. (1975). The meaning of "meaning." In H. Putnam, Mind, language, and reality: Philosophical papers (Vol. 2, pp. 215-271). Cambridge: Cambridge University Press.

Rosch, E., Mervis, C. B., Gray, W. D., Johnson, D. M., \& BoyesBraEm, P. (1976a). Basic objects in natural categories. Cognitive Psychology, 8, 382-439.

Rosch, E., Mervis, C. B., Gray, W. D., Johnson, D. M., \& BoyesBraem, P. (1976b). Basic objects in natural categories (Working Paper 43). Berkeley: University of California, Language Behavior Research Laboratory.

SLOMAN, S. A. (1996). The empirical case for two systems of reasoning. Psychological Bulletin, 119, 3-22.

Sloman, S. A., Love, B. C., \& AhN, W. (1998). Feature centrality and conceptual coherence. Cognitive Science, 22, 189-228.

TVERSKy, A. (1977). Features of similarity. Psychological Review, 84, 327-352.

TVersKy, A., \& KahNeman, D. (1973). Availability: A heuristic for judging frequency and probability. Cognitive Psychology, 4, 207-232.

TVERSKY, B., \& HEMENWAY, K. (1984). Objects, parts, and categories. Journal of Experimental Psychology: General, 113, 169-193.

Wittgenstein, L. (1953). Philosophical investigations. New York: MacMillan.

\section{NOTES}

1. Because no intermediate-level feature seemed to have high category validity for knit shirt, we used the feature covers the torso.

2. A 1-5 scale was used with Brown students.

3. In the same experiment, the participants also drew the dependency structure of basic and superordinate categories in a blocked, counterbalanced design. This part of the experiment is not relevant for the purpose of this paper. There was no effect of order, and hence this part of the experiment can be safely ignored.

4. Because we did not have dependency values for the intermediatelevel feature of knit shirt (see note 1), these analyses did not include that category. The same conclusions hold if the analysis is done using any intermediate-level feature of knit shirt appearing in the Appendix. 
APPENDIX

Categories Used in Experiment 2 and

Category Features Used in Experiment 3

\begin{tabular}{|c|c|c|}
\hline Category & Feature & Level of Feature \\
\hline Green seedless grapes & $\begin{array}{l}\text { are edible } \\
\text { have seeds } \\
\text { are sweet } \\
\text { have bunch } \\
\text { are juicy } \\
\text { can make wine with } \\
\text { are grown in vine } \\
\text { are green } \\
\text { are small }\end{array}$ & $\begin{array}{l}\text { Superordinate } \\
\text { Superordinate } \\
\text { Superordinate } \\
\text { Intermediate level } \\
\text { Intermediate level } \\
\text { Intermediate level } \\
\text { Intermediate level } \\
\text { Specific } \\
\text { Specific }\end{array}$ \\
\hline Knit shirt & $\begin{array}{l}\text { can be worn } \\
\text { keeps people warm } \\
\text { has buttons } \\
\text { has cuffs } \\
\text { is made of cotton } \\
\text { has a pocket } \\
\text { has collar } \\
\text { stretches } \\
\text { is comfortable }\end{array}$ & $\begin{array}{l}\text { Superordinate } \\
\text { Superordinate } \\
\text { Intermediate level } \\
\text { Intermediate level } \\
\text { Intermediate level } \\
\text { Intermediate level } \\
\text { Intermediate level } \\
\text { Specific } \\
\text { Specific }\end{array}$ \\
\hline Claw hammer & $\begin{array}{l}\text { fixes things } \\
\text { makes things } \\
\text { is made of metal } \\
\text { is used to nail } \\
\text { has a handle } \\
\text { has a wooden handle } \\
\text { has a head } \\
\text { has a metal head } \\
\text { pulls out nails }\end{array}$ & $\begin{array}{l}\text { Superordinate } \\
\text { Superordinate } \\
\text { Superordinate } \\
\text { Intermediate level } \\
\text { Intermediate level } \\
\text { Intermediate level } \\
\text { Intermediate level } \\
\text { Intermediate level } \\
\text { Specific }\end{array}$ \\
\hline Cucumber & $\begin{array}{l}\text { does not move around } \\
\text { is found in soil } \\
\text { has chlorophyll } \\
\text { would die without light } \\
\text { has roots } \\
\text { has seeds } \\
\text { is usually silent } \\
\text { can be harvested } \\
\text { is commonly eaten } \\
\text { is solid to the touch } \\
\text { has a rind } \\
\text { is green } \\
\text { has bright colors } \\
\text { is crunchy if chewed } \\
\text { is eaten for snack } \\
\text { has a pleasant taste } \\
\text { is smooth }\end{array}$ & $\begin{array}{l}\text { Superordinate } \\
\text { Superordinate } \\
\text { Superordinate } \\
\text { Superordinate } \\
\text { Superordinate } \\
\text { Superordinate } \\
\text { Superordinate } \\
\text { Intermediate level } \\
\text { Intermediate level } \\
\text { Intermediate level } \\
\text { Specific } \\
\text { Specific } \\
\text { Specific } \\
\text { Specific } \\
\text { Specific } \\
\text { Specific } \\
\text { Specific }\end{array}$ \\
\hline Robin & $\begin{array}{l}\text { has toes } \\
\text { is warm-blooded } \\
\text { can think } \\
\text { hunts for its prey } \\
\text { can walk } \\
\text { has a tail } \\
\text { actively seeks food } \\
\text { has few offspring } \\
\text { reproduces sexually } \\
\text { breathes air } \\
\text { is mobile } \\
\text { can sing } \\
\text { can fly }\end{array}$ & $\begin{array}{l}\text { Superordinate } \\
\text { Superordinate } \\
\text { Superordinate } \\
\text { Superordinate } \\
\text { Superordinate } \\
\text { Superordinate } \\
\text { Superordinate } \\
\text { Superordinate } \\
\text { Superordinate } \\
\text { Superordinate } \\
\text { Superordinate } \\
\text { Intermediate level } \\
\text { Intermediate level }\end{array}$ \\
\hline
\end{tabular}




\section{APPENDIX (Continued)}

\begin{tabular}{ll} 
is a carnivore & Intermediate level \\
makes distinct vocalizations & Intermediate level \\
has a beak & Intermediate level \\
can lay eggs & Intermediate level \\
has wings & Intermediate level \\
builds nests & Intermediate level \\
has two legs & Intermediate level \\
has feathers & Intermediate level \\
is swift & Specific \\
is red & Specific \\
has bright colors & Specific \\
is found in rural areas & Specific \\
does not move around & Superordinate \\
has seeds & Superordinate \\
gets its nutrients via roots & Superordinate \\
is usually silent & Superordinate \\
would die without light & Superordinate \\
breathes air & Superordinate \\
has chlorophyll & Superordinate \\
is found in soil & Superordinate \\
has leaves & Intermediate level \\
is used for construction & Intermediate level \\
has a bark & Intermediate level \\
is rough to the touch & Intermediate level \\
is green & Intermediate level \\
is large in size & Intermediate level \\
has branches & Intermediate level \\
is solid to the touch & Intermediate level \\
has pointy needles & Specific \\
has a strong odor & Specific \\
\hline & \\
\hline & \\
\hline
\end{tabular}

(Manuscript received October 8, 1997; revision accepted for publication June 30, 1998.) 